\title{
Evaluation of the Side Effect Profile of Intracavitary Bacillus Calmette-Guérin Treatment in Non-muscle-invasive Bladder Cancer
}

\author{
(D) Şenol Tonyalı MD, (D) Mustafa Karaaslan MD, (D) Mehmet Yılmaz MD, (D) Cavit Ceylan MD \\ Türkiye Yüksek Ihtisas Training and Research Hospital, Clinic of Urology, Ankara, Turkey
}

\begin{abstract}
Objective: To reveal the association of side effect profiles seen in patients receiving intravesical bacillus Calmette-Guérin (BCG) immunotherapy for non-muscle-invasive bladder cancer (NMIBC) treatment with patient age and possible changes over time due to the use of different BCG strains.

Materials and Methods: We retrospectively analyzed the medical records of all patients who received BCG for NMIBC in our hospital between January 2013 and December 2017. Investigated parameters included patients' demographics, treatment dates, local and systemic side effects (dysuria, hematuria, cystitis, headache, arthralgia/myalgia, chills, fever, fatigue, epididymo-orchitis, renal abscess, pneumonia, hepatitis, and sepsis), need for hospitalization and antituberculous therapy, discontinuation of therapy, and doses received.

Results: The study included 89 patients ( 84 male, 5 female) with a mean age of $67.4 \pm 10.2$ years. The most common side effects were dysuria $(41.6 \%)$, chills $(31.5 \%)$, hematuria $(30.3 \%)$, fever $(29.2 \%)$, cystitis $(24.7 \%)$, and fatigue $(23.6 \%)$. Ten patients $(11.2 \%)$ were hospitalized due to treatment-related side effects. Ten patients discontinued treatment due to side effects. Sixty-two patients (69.7\%) experienced at least 1 local or systemic side effect. There was no significant difference between patients younger and older than 70 years in terms of side effect rates (69.8\% vs $69.4 \%, p=0.576$ ). In addition, a significant difference was not observed in complication rates when we compared treatment before and after 2016 (65.3\% vs $75 \%, p=0.322)$.

Conclusion: Intracavitary BCG can be the treatment of choice in NMIBC even in patients at an advanced age. The absence of a significant change in complication rates over the years despite changing strains may be evidence that strains have a similar side effect profile.
\end{abstract}

Keywords: Bladder cancer, bacillus Calmette-Guérin, strain, intracavitary

\section{Introduction}

Bladder cancer is the eleventh most common cancer globally and the $7^{\text {th }}$ most common among men. Most bladder cancers are of urothelial cancer histology, and these are categorized as either muscle-invasive bladder cancer (MIBC) or non-muscleinvasive bladder cancer (NMIBC). NMIBC accounts for about $75 \%$ of all bladder cancers (1). NMIBC has a recurrence rate of $50-80 \%$ and progresses to MIBC in about $30 \%$ of cases. NMIBCs are candidates for bladder-sparing treatment approaches (2).
Various intravesical chemotherapy and immunotherapy options are available for patients with intermediate- to high-risk NMIBC. Bacillus Calmette-Guérin ( $B C G$ ) is a heterogeneous organism, with at least 8 different strains used for intravesical immunotherapy (3). It was first defined in 1976 by Morales et al. (4) and is classically administered based on a 6-week induction schedule. Optimal maintenance dose and interval have not been definitely determined, but durations of 1 to 3 years have been reported (5). BCG therapy is superior to intravesical chemotherapeutics in reducing the risk of both recurrence and progression (3). 
Although BCG immunotherapy is proven effective for NMIBC, it may also cause several local and systemic side effects. These adverse reactions can range from mild malaise and fever to fatal or life-threatening sepsis (6).

In our clinic, BCG Culture SSI (Danish strain 1331, $120 \mathrm{mg}$ ) has been used most commonly for intracavitary BCG therapy since mid-2015. Prior to that, the BCG Connaught strain $(81 \mathrm{mg}$ ) was preferred.

In this study, we evaluated patients who received intravesical BCG immunotherapy for NMIBC to determine whether side effect profiles are associated with patient age and whether they show variation over time due to the use of different strains.

\section{Materials and Methods}

The data of patients who received intracavitary BCG treatment in our hospital for intermediate- to high-risk NMIBC between January 2013 and December 2017 were examined retrospectively. Patients who reported any local or systemic side effects during induction or maintenance BCG therapy were noted. Patients who had previously received chemotherapy or radiotherapy for bladder tumor were excluded from the study. Urine analysis and urine culture were conducted prior to BCG therapy in all cases. Treatment was postponed for patients with hematuria and active urinary tract infection. Induction therapy was administered weekly for 6 weeks; maintenance therapy was typically given as 3 weekly doses at 3, 6, 12, 18, 24, 30 and 36 months. Analyzed parameters included the patients' demographic data, dates of treatment, local and systemic side effects (dysuria, hematuria, cystitis, headache, arthralgia/ myalgia, chills, high fever, malaise, epididymo-orchitis, renal abscess, pneumonia, hepatitis, sepsis), need for hospitalization, need for antituberculous therapy, treatment discontinuation due to side effects, and dosage received. All patients with available data were included in the study, regardless of gender or age.

Patients were divided into 2 groups based on age. Patients under 70 years of age and those older than 70 years of age were compared in terms of side effect profile. In addition, patients treated before and after 2016 were compared to determine whether the change of strains had an influence on the side effect profile over time.

Ethics committee approval was obtained from Türkiye Yüksek Ihtisas Training and Research Hospital Board of Education Planning and Coordination. Written informed consent was not obtained because this study is retrospective.

\section{Statistical Analysis}

The data were analyzed using IBM SPSS Statistics for Mac v.21.0 (IBM Corp., Armonk, NY). Quantitative values were expressed as mean \pm standard deviation and qualitative values were expressed in numbers and percents. Comparisons between groups were done using Mann-Whitney $U$ test and chi-square test. $\mathrm{P}<0.05$ was considered statistically significant.

\section{Results}

A total of 89 patients ( 84 males and 5 females) were included in the study. Their mean age was $67.4 \pm 10.2$ years. Mean ages of the males and females in the study were $67.8 \pm 10.1$ years and $60.8 \pm 10.7$ years, respectively. There was no significant difference between males and females with regard to age $(p=0.137)$. The most common side effects were dysuria (41.6\%), chills (31.5\%), hematuria $(30.3 \%)$, high fever $(29.2 \%)$, cystitis $(24.7 \%)$, and malaise $(23.6 \%)$. One patient $(1.1 \%)$ was diagnosed with epididymo-orchitis, 1 (1.1\%) with renal abscess, 1 (1.1\%) with pneumonia, and 2 (2.2\%) with hepatitis related to treatment. A total of 4 patients (3.4\%) received antituberculous therapy. Ten patients (11.2\%) were hospitalized due to BCGrelated side effects. Ten patients $(11.2 \%)$ had to discontinue intracavitary BCG therapy due to side effects. Of the 10 patients who discontinued treatment, 6 were receiving induction and 4 were receiving maintenance doses. Patients who were unable to complete the induction treatment were switched to intracavitary chemotherapeutic agents. The mean age of the hospitalized patients was $70.2 \pm 8.4$ years, whereas that of the other patients was $67 \pm 10.4$ years $(p=0.366)$. Sixty-two patients (69.7\%) experienced 1 or more systemic or local side effect. There was no difference between patients over and under the age of 70 in the rate of side effects $(69.4 \%$ vs $69.8 \%, p=0.576)$. There was also no significant difference in rate of side effects between patients treated before and after the year 2016 (65.3\% vs $75 \%, p=0.322$ ) (Table 1 ).

\section{Discussion}

Intracavitary BCG immunotherapy for NMIBC was first described by Morales et al. (4) and it was reported that certain criteria must be met for effective BCG immunotherapy. These include the ability to develop immune response against mycobacterial antigens, sufficient number of live bacilli, close contact between BCG and tumor, relatively low tumor burden, and absence of major systemic side effects (4).

There is no consensus on the optimal BCG preparation, dose, or administration schedule. Although BCG toxicity is commonly reported after intense regimens, very severe side effects may also be seen after only a few instillations. In a study conducted with 2602 patients, fever was reported in 2.9\%, pneumonia/ hepatitis in $0.7 \%$, gross hematuria in 1\%, arthralgia in $0.5 \%$ and epididymo-orchitis in $0.4 \%$. No variation was observed in the complication rates of different BCG strains (6). It was shown in another study that decreasing the BCG dose did not reduce the side effect rate (7). Although the rates of hematuria and fever reported in our current study were lower than those obtained in the aforementioned one, the rates of pneumonia, hepatitis, and epididymo-orchitis ratios were similar. There was also no significant change in the complication rates of patients receiving $B C G$ over time. This may be evidence that different strains have similar side effect profiles.

Various solutions have been attempted to minimize the related side effects without compromising the therapeutic efficacy of BCG therapy. These include gradually reducing the dose given in each successive instillation or extending the intervals between instillations. It was reported that common side effects occurred significantly less frequently in patients who received a reduced dose of $75 \mathrm{mg}$ instead of the standard $150 \mathrm{mg}$ dose of BCG (Pasteur strain) [cystitis, $57 \%$ to $32 \%$; fever $<38.5{ }^{\circ} \mathrm{C}$, $26 \%$ to $12 \%$; gross hematuria $24 \%$ to $13 \%(p<0.05)](8)$. In another study by Irie et al. (9), 40 mg BCG (Tokyo 171 strain), which is half the full dose, had similar efficacy to the full dose but resulted in a significant reduction of BCG toxicity. 
Tonyalı et al.

Side Effect Profile of Intracavitary Bacillus Calmette-Guérin Treatment

\begin{tabular}{|c|c|c|c|c|c|c|c|}
\hline \multirow{2}{*}{\multicolumn{2}{|c|}{ Side effects }} & \multicolumn{3}{|l|}{ Age (years) } & \multicolumn{3}{|l|}{ Treatment year } \\
\hline & & $<70(\mathrm{n}=53)$ & $>70(n=36)$ & $\mathbf{p}$ & 2016 and later $(n=40)$ & Before $2016(n=49)$ & $\mathbf{p}$ \\
\hline \multirow{5}{*}{ Systemic side effects } & Fever & $15(28.3 \%)$ & $11(30.6 \%)$ & 0.817 & $14(35 \%)$ & $12(24.5 \%)$ & 0.350 \\
\hline & Arthralgia/myalgia & $4(7.5 \%)$ & $6(16.7 \%)$ & 0.181 & $3(7.5 \%)$ & $7(14.3 \%)$ & 0.313 \\
\hline & Renal abscess & $1(1.9 \%)$ & 0 & 0.407 & 0 & $1(2 \%)$ & 0.364 \\
\hline & Pneumonia & 0 & $1(2.8 \%)$ & 0.222 & $1(2.5 \%)$ & 0 & 0.266 \\
\hline & Hepatitis & $1(1.9 \%)$ & $1(2.8 \%)$ & 0.781 & 0 & $2(4.1 \%)$ & 0.196 \\
\hline Local side effects & Dysuria & $24(45.3 \%)$ & $13(36.1 \%)$ & 0.511 & $18(45 \%)$ & $19(38.8 \%)$ & 0.666 \\
\hline Hospitalization & - & $6(11.3 \%)$ & $4(11.1 \%)$ & 0.975 & $7(17.5 \%)$ & $3(6.1 \%)$ & 0.091 \\
\hline Antituberculous therapy & - & $1(1.9 \%)$ & $2(5.6 \%)$ & 0.347 & 0 & $3(6.1 \%)$ & 0.111 \\
\hline $\begin{array}{l}\text { Treatment discontinuation } \\
\text { due to side effects }\end{array}$ & - & $4(7.5 \%)$ & $6(16.7 \%)$ & 0.181 & $4(10 \%)$ & $6(12.2 \%)$ & 0.739 \\
\hline
\end{tabular}

In still another study, Koga et al. (10) reported a $95.9 \%$ incidence of side effects with general intracavitary BCG (Tokyo 172 strain). Administration of BCG for treatment purposes instead of prophylaxis and BCG dose were found to be independent risk factors for side effects (10). In another report, instillation of BCG (Connaught strain) doses reduced from 81 $\mathrm{mg}$ to $27 \mathrm{mg}$ had similar effects on progression as the standard dose, with less toxicity (11). In a study including 1316 patients, Brausi et al. (5) demonstrated that neither dose nor treatment duration had a significant influence on the side effect profile of intracavitary BCG treatment. Making a similar comparison was not possible in our study since we had patients receiving both induction and maintenance treatment and sufficient data regarding progression were not available.

In a study conducted by Heiner and Terris (12) with 58 patients receiving intracavitary $B C G, 22(37.9 \%)$ of the patients had treatment-related complications. Complication rates in patients receiving maintenance treatment were $17.6 \%$ for those less than 70 of age and $48.6 \%$ for those over 70 . The mean age of patients with complications was significantly higher than those without complications (76 years vs 70.3 years, $p<0.00001$ ) (12). In our study group there was no significant difference in rates of complications related to intracavitary BCG therapy between patients under and over the age of 70 .

\section{Study Limitations}

Our study included a relatively small number of patients. Due to lack of patient cooperation and insufficient record-keeping, the data were not clear in terms of which patient received which strain how many times, and after how many doses side effects started or ended. Moreover, although the induction treatment schedule was the same, the maintenance treatment protocol may vary between clinicians.

\section{Conclusion}

Intracavitary BCG treatment is a treatment option for NMIBC that can also be used in patients at advanced ages. Despite strain variations, complication rates did not change significantly over the years in our study, suggesting that strains have similar side effect profiles.

\section{Ethics}

Ethics Committee Approval: Approval was obtained from Türkiye Yüksek İhtisas Training and Research Hospital Board of Education Planning and Coordination.

Informed Consent: Not obtained because this study is retrospective.

Peer-review: Externally peer-reviewed.

\section{Authorship Contributions}

Surgical and Medical Practices: Ş.T., M.K., M.Y., C.C., Concept: S..T., C.C., Design: Ş.T., M.K., M.Y., C.C., Data Collection or Processing: Ş.T., M.K., M.Y., C.C., Analysis or Interpretation: Ş.T., M.K., M.Y., C.C., Literature Search: Ş.T., M.K., M.Y., C.C., Writing: Ş.T.

Conflict of Interest: No conflict of interest was declared by the authors.

Financial Disclosure: The authors declared that this study received no financial support.

\section{References}

1. Woldu SL, Bagrodia A, Lotan Y. Guideline of guidelines: non-muscleinvasive bladder cancer. BJU Int 2017;119:371-380.

2. Gregg JR, Dahm P, Chang SS. Guideline-based management of nonmuscle invasive bladder cancer. Indian J Urol 2015;31:320-326.

3. Chang SS, Boorjian SA, Chou R, et al. Diagnosis and Treatment of Non-Muscle Invasive Bladder Cancer: AUA/SUO Guideline. J Urol 2016;196:1021-1029. 
4. Morales A, Eidinger D, Bruce AW. Intracavitary Bacillus CalmetteGuerin in the treatment of superficial bladder tumors. J Urol 1976;116:180-183.

5. Brausi M, Oddens J, Sylvester R, et al. Side effects of Bacillus Calmette-Guerin (BCG) in the treatment of intermediate- and highrisk Ta, T1 papillary carcinoma of the bladder: results of the EORTC genito-urinary cancers group randomised phase 3 study comparing one-third dose with full dose and 1 year with 3 years of maintenance BCG. Eur Urol 2014;65:69-76.

6. Lamm DL, van der Meijden PM, Morales A, et al. Incidence and treatment of complications of bacillus Calmette-Guerin intravesical therapy in superficial bladder cancer. J Urol 1992;147:596-600.

7. Galvan L, Ayani I, Arrizabalaga MJ, Rodriguez-Sasiain JM. Intravesical BCG therapy of superficial bladder cancer: study of adverse effects. J Clin Pharm Ther 1994;19:101-104.

8. Bassi P, Spinadin R, Carando R, et al. Modified induction course: a solution to side-effects? Eur Urol 2000;37(Suppl 1):31-32.
9. Irie A, Uchida T, Yamashita $H$, et al. Sufficient prophylactic efficacy with minor adverse effects by intravesical instillation of low-dose bacillus Calmette-Guerin for superficial bladder cancer recurrence. Int J Urol 2003;10:183-189.

10. Koga $H$, Kuroda $M$, Kudo $S$, et al. Adverse drug reactions of intravesical bacillus Calmette-Guerin instillation and risk factors of the development of adverse drug reactions in superficial cancer and carcinoma in situ of the bladder. Int J Urol 2005;12:145-151.

11. Martinez-Pineiro JA, Martinez-Pineiro L, Solsona E, et al. Has a 3-fold decreased dose of bacillus Calmette-Guerin the same efficacy against recurrences and progression of $\mathrm{T} 1 \mathrm{G} 3$ and Tis bladder tumors than the standard dose? Results of a prospective randomized trial. J Urol 2005; 174:1242-1247.

12. Heiner JG, Terris MK. Effect of advanced age on the development of complications from intravesical bacillus Calmette-Guerin therapy. Urol Oncol 2008;26:137-140. 